\title{
Identical Ovarian and Deep Pelvic Endometriosis with Colorectal Involvement in Monozygotic Twins: A Case Report and Review of the Literature
}

\author{
Adel Shervin*1,Fariba Behnia-Willison ${ }^{2}$ and Joseph Miller ${ }^{3}$ \\ ${ }^{1}$ FBW Gynaecology Plus, Flinders University Adelaide, Australia \\ ${ }^{2}$ Department of Obstetrics, Gynecology and MIS, Farmanieh Hospital, Tehran, Iran \\ ${ }^{3}$ Department of Obstetrics, Gynecology and Minimally invasive surgery (MIS), Farmanieh Hospital, Tehran, Iran
}

Received: October 30,2017; Published: November 08, 2017

*Corresponding author: Adel Shervin, FBW Gynaecology Plus, Flinders University Adelaide, Australia; Email: adelshervin@gmail.com

\begin{abstract}
Endometriosis is a common benign gynecologic disease characterised by the presence of ectopic endometrial tissue outside the uterus. We present a brief review on the genetic factors underlying endometriosis, followed by a case report on concordant anatomical distribution of deep infiltrating endometriosis (DIE) in a pair of monozygotic (MZ) twins. To our knowledge, this is one of the first reported cases of DIE in MZ twins. The remarkable concordance and resemblance of deep disease involving the same anatomical sites, ovaries, pelvic floor and rectosigmoid colon in our MZ twins reiterates the role and impact of genetic factors in the pathogenesis of endometriosis.
\end{abstract}

Keywords: Deep Infiltrating Endometriosis; Sigmoid Resection; Monozygotic Twins; Genetics

Abbreviations: MZ: Mono Zygotic; DZ: Di Zygotic; DIE : Deep Infiltrating Endometriosis; IVP: Intravenous Pyelogram; TVS: Transvaginal Ultrasound; RES: Rectal Endoscopic Sonography; SMM: Smooth Muscle Metaplasia

\section{Introduction}

Endometriosis is a polygenic multi factorial disease. Incidence of deep infiltrating endometriosis (DIE) involving the GI tract is estimated at $8-12 \%[1,2]$ and commonly involves the rectosigmoid colon. Endometriosis is one of the most common benign gynecologic diseases. It causes pelvic pain and sub fertility, and is characterised by the presence of ectopic endometrial tissue outside the uterus [3]. Endometriosis is under-diagnosed and associated with a mean latency of 6.7 years from onset of symptoms to definitive diagnosis [4]. Most estimates of prevalence are made on the basis of surgical cases or small samples, and are highly selective, ranging between $5 \%$ and $10 \%$ in women of reproductive age, and up to $50 \%$ among infertile women [5-7]. Endometriosis has an important socio-economic impact, because it greatly lowers quality of life for a significant portion of the population, and is responsible for substantial health expenditure, diagnosis, treatment, and loss of economic performance. The cost of endometriosis to the US health care system was $\$ 69.4$ billion in 2009 [8]. Despite 150 years of hypothesis-driven research, the cause of endometriosis remains uncertain. Therapeutic options are therefore limited, often lacking unanimous consensus. However, there is mounting evidence that endometriosis is a complex multifactorial disease, with both genetic and environmental components contributing to disease susceptibility [7]. In 1980, Simpson et al. published the first formal genetic study of endometriosis [9]. Studying 123 probands with histologically proven endometriosis, they found that $5.9 \%$ of female siblings over the age of 18 years had endometriosis; the mothers were affected in $8.1 \%$ of cases.

However, only $1 \%$ of the patients' husband's first-degree relatives (controls) had the disease. Women with an affected sibling or parent were more likely to have a severe form of endometriosis [10]. Severe endometriosis was present in $61 \%$ of probands who had an affected first-degree relative, whereas it was only present in $23 \%$ of the affected probands with no affected first-degree relatives. One recent meta-analysis combining results from a genome-wide association study and replication studies showed that of the nine loci found to be associated with endometriosis in at least one of the studies, six remained statistically significant genome-wide, and two showed borderline statistically significant 
genome-wide association with moderate/severe disease [11]. In an Australian twin-based study, a twofold increase in endometriosis risk in monozygotic (MZ) compared with dizygotic (DZ) twin pairs was reported [7], which suggests that the genetic component contributing to phenotypic variability in endometriosis is about $52 \%$. These data imply that endometriosis is a complex genetic trait, and indicate that a number of genes interact with each other to form disease susceptibility, with the phenotype emerging in the presence of environmental risk factors which in themselves account for $53 \%$ of disease liability. Environmental chemicals, as well as food, have been discussed as possible contributing factors [12-14]. However, there is no existing evidence as to the nature of this environmental contribution. Only one study to date has used quantitative analysis to examine the contribution of genetic and environmental factors to endometriosis, using a small twin sample [7]. A larger twin sample is expected to provide further clarification on the role of genetic and environmental factors [12-14]. In this report, we present a case of deep infiltrating endometriosis (DIE) in a pair of monozygotic (MZ) twins.

\section{Materials and Methods}

A pair of monozygotic twins was referred to our office within the same year, mainly due to severe chronic pelvic pain and infertility, with the following pertinent clinical information.

a) Twin A : A 31-year-old nulliparous woman was admitted to Farmanieh Hospital (Tehran, Iran) complaining of heavy menstrual blood loss, progressive chronic severe pelvic pain and dyspareunia for the past six years, painful defecation with passage of narrow, occasionally blood-tinged stool, and history of failed hormonal medical treatment on and off during the past six years. The patient had been infertile for the past two years. She had a history of hypothyroidism, thalassemia minor, cervical spinal cord tumour surgery, rhinoplasty and eye surgery. Transvaginal ultrasound detected a $25 \times 22 \mathrm{~mm}$ cyst in the right ovary and two heterogeneous hypoechoic foci $(32 \times 21 \mathrm{~mm}$ and $29 \times 18 \mathrm{~mm}$ respectively) in the left ovary; these observations were compatible with endometrioma. Colonoscopy results were negative. Hemoglobin level was 11.4g/dL, serum CA 125 level was $60.67 \mathrm{U} / \mathrm{mL}$, serum CA 19-9 level was $6.4 \mathrm{U} / \mathrm{mL}$, and AMH level was $5.7 \mathrm{ng} / \mathrm{mL}$. Intravenous pyelogram (IVP) results were negative.Magnetic Resonance Imaging (MRI) revealed two T1 foci in both ovaries $(25 \times 15 \mathrm{~mm}$ and $20 \times 10 \mathrm{~mm}$ high respectively), which was suggestive of a hemorrhagic cyst or, more probably, a dermoid cyst. No other pathology was noted, including for the intestinal tract.

b) Twin B: A 31-year-old nulliparous woman was admitted to Farmanieh Hospital (Tehran, Iran) complaining of menorrhagia, progressive severe pelvic pain with rectal radiation for six years, painful defecation with occasional blood-tinged stool, severe dyspareunia for the past two years and failed hormonal medical treatment for the past six years. She had a history of hypothyroidism, thalassemia minor, mitral valve prolapse rhinoplasty and eye surgery. Two transvaginal pelvic ultrasounds revealed a small anterior wall myoma; there were no other findings. Hemoglobin level was $10.8 \mathrm{~g} / \mathrm{dL}$, serum CA 125 level was 21U/mL, serum CA 19-9 level was 9.4 $\mathrm{U} / \mathrm{mL}$, and AMG level was $7.4 \mathrm{ng} / \mathrm{mL}$. IVP results were within normal limits. MRI of the pelvis detected three small myomas $(10-15 \mathrm{~mm})$ at the anterior uterine wall and a $20 \mathrm{~mm}$ follicle in the left ovary. There were no other findings, including for the bowel. Colonoscopy results were within normal limits. A double-contrast barium enema detected segmental luminal narrowing with upward displacement of the sigmoid area, with mucosal thinning. These findings suggested extrinsic pressure, mostly due to endometriosis, which seemed to have involved the posterior wall of the sigmoid colon.

\section{Results}

\section{Twin A}

A laparoscopic segmental sigmoid resection was performed, with staple re-anastomosis, resection of pelvic floor DIE, and resection of a bilateral endometrioma. The procedure took 152 minutes. The patient was discharged after four days shown in below Figure 1.
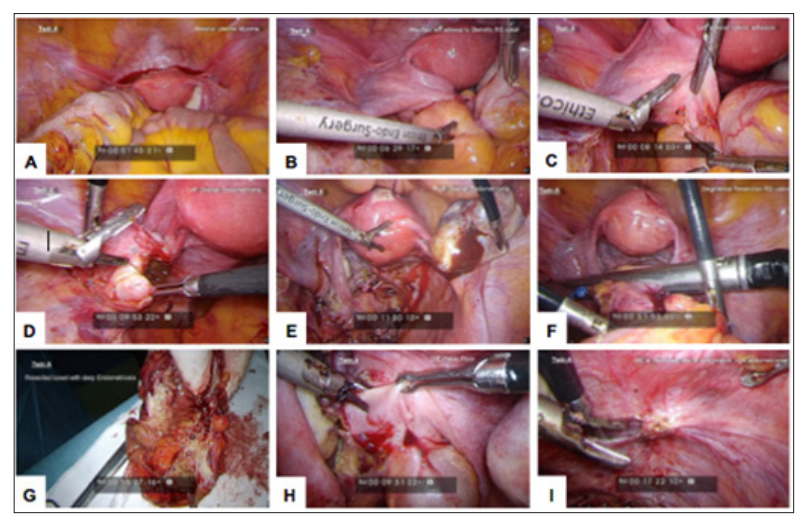

Figure 1: Laparoscopic images for Twin A.
(A) Anterior uterine myoma,
(B) Attached left adnexa to stenotic RS colon,
(C) Left adnexal colonic adhesion,
(D) Left ovarian endometrioma,
(E) Right ovarian endometrioma,
(F) Segmental resection of RS colon,
(G) Resected bowel with deep endometriosis,
(H) DIE pelvic floor,and
(I) DIE at peritoneal site of invagination.

\section{Twin B}

Twin B's initial procedure was performed in the same month as Twin A's procedure. Laparoscopic adhesiolysis was performed, with resection of pelvic floor DIE, resection of ovarian endometrioma, and shaving of rectal deep endometriosis. Bowel resection was deferred because of inadequate bowel preparation, and laparoscopic sigmoid resection with staple anastomosis was performed 8 weeks after the initial procedure (Figure 2).

\section{Discussion}

Anatomical involvement of the relevant pelvic organ in this pair of identical twins was nearly identical. The twins had grown up in the same environment all their lives, both worked as secretaries at the same institution, and both were experiencing 
not only the symptoms of DIE, but also signs and symptoms of intestinal involvement. The ultrasound and MRI for both patients was reported negative, which clearly indicates the importance of experienced radiologists for the detection of deep endometriosis [15], and reiterates the importance of bimanual exam by clinicians, particularly at the time of menstruation, to achieve an accurate diagnosis,. In fact, available data clearly indicates that the accuracy of good physical examination (PE) is not much different from that of transvaginal ultrasound (TVS), rectal endoscopic sonography (RES) and MRI, all within the $80 \%$ range [16]. Twin A was managed by laparoscopic segmental sigmoid resection and staple reanastomosis, along with global resection of DIE from the pelvic floor and endometriomas. We were intending to do the same for Twin B, but in OR the lack of adequate bowel preparation was noted; thus bowel resection was postponed and was performed 8 weeks later under adequate bowel preparation.
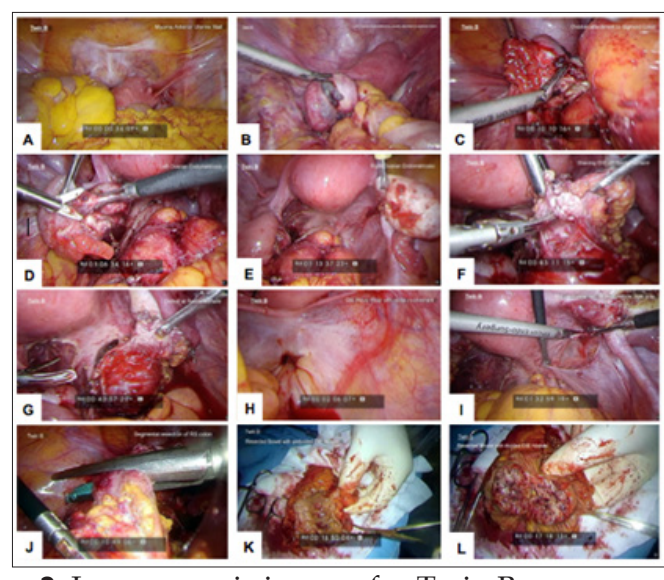

Figure 2: Laparoscopic images for Twin B.

(A) Myoma at anterior uterine wall,

(B) Left ovarian endometrioma severely attached to sigmoid colon,

(C) Ovarian attachment to sigmoid colon,

(D) Left ovarian endometriosis,

(E) Right ovarian endometriosis,

(F) Shaving DIE off rectal surface,

(G) Defect at rectal surface,

(H) DIE pelvic floor with rectal involvement,

(I) DIE peritoneal site on invagination, right side,

(J) Segmental resection of RS colon,

(K) Resected bowel with undivided DIE nodule and

(L) Resected bowel with divided DIE nodule.

However, after resection of endometriomas and pelvic floor deep lesions, larger DIE at the posterior cervix with extension to the rectum was managed by laparoscopic shaving, and the defect was closed in two layers. No intraoperative or postoperative complications were encountered in either twin. In many instances, the culprit for DIE is endometrioma, which starts from its peritoneal site of invagination (Figure 3). By extension medially, it involves the parametrium, rectum, recto-vaginal space, and, by deeper extension, the ureter and hypogastric nerve may also be involved. Thus, timely and proper surgical management of endometrioma and excision of its dependent peritoneal site could serve not only to manage patients' pain, but also to conceivably prevent a significant percentage of DIE. In one study focusing exclusively on patients with rectosigmoid lesions, $48 \%$ and $84 \%$ had ovarian endometriosis and retrocervical lesions, respectively [17] (Figure 3).

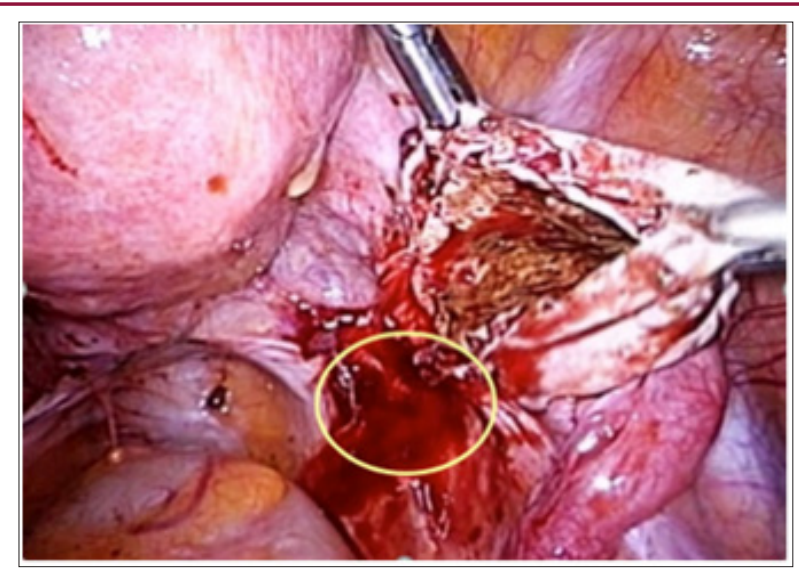

Figure 3: Endometrioma and the peritoneal site of invagination.

Both superficial peritoneal and ovarian endometriomas may be found in association with DIE in variable percentages, thus contributing to the intensity of painful stimuli, as well as to infertility [18]. Thisraises the question of whether DIE is an independent form of the disease, or whether it represents its most severe clinical representation [19]. Nisolle and Donnez have argued that peritoneal endometriosis, ovarian endometriosis and adenomyotic nodules of the rectovaginal septum are three different entities [20]. In a retrospective observational study by Kamergorodsky [21]. Involving 176 patients and 271 biopsies, the histologic differentiation in superficial endometriosis, DIE and ovarian endometriomas was evaluated. Results showed a predominance of the undifferentiated glandular pattern $(33.5 \%)$ and mixed glandular pattern $(46.9 \%)$ in deeply infiltrating endometriosis lesions, whereas the welldifferentiated glandular pattern $(41.8 \%)$ was most frequently seen in superficial endometriosis lesions, and in ovarian endometriomas a predominance of both the undifferentiated (40.5\%) and mixed patterns (37.8\%) was observed. There was no significant histologic difference between infiltrative endometriosis of the uteroscacral ligaments, recto-vaginal endometriosis, or bowel endometriosis. There was a predominance of the well-differentiated pattern in more superficial lesions and the undifferentiated pattern in deeper lesions, suggesting a similar mechanism to explain the invasive potential of endometriosis.

Recently, Sapkota [22] analysed genetic risk scores derived from two large European genome-wide association (GWA) datasets from a previous multi-ethnic GWA meta-analysis of endometriosis. They found that genetic factors contributing to minimal disease might differ from those contributing to more severe endometriosis, and that more severe endometriosis cases exhibit greater genetic loading than minimal or mild disease. The genetic burden generally increased from less severe (minimal) to more severe disease, consistent with disease progression. Considering the fact that endometriotic lesions undergo repeated cyclic bleeding (injury) and repair like other organs leading to fibrosis [23], it has been suggested that endometriotic lesions are fundamentally wounds with repeated tissue injury resulting in smooth muscle metaplasia 
(SMM) and ultimately fibrosis, which somehow do not heal like an ordinary wound [24]. This points to the complex microenvironment and cross-reaction of endometrioc lesions with other cells, i.e. platelets and macrophages, their gradual but progressive evolution to SMM and fibrosis and, on occasion, to malignancy and the new traits/phenotypes they may acquire while losing old ones. This dynamic feature of endometriotic lesions may explain the reason for some conflicting results in this area of research, including the fact that development of biomarkers for the diagnosis or prognosis of endometriosis has posed a challenge, at least until now.

Endometriosis appears to be a progressive disease, as evidenced by longitudinal laparoscopies in female baboons [25]. There are at least eight studies reported (with a total of 162 patients) on repeat laparoscopy in women assigned to placebo treatment. The results indicate nearly equal distribution among women whose disease stage deteriorated (31\%), was unchanged $(32 \%)$, or improved $(38 \%)$. In fact, all but one study noted that $23 \%$ of placebo patients had complete regression of the disease over intervals of 4 to 39 months, meaning that in over two thirds of women the disease will either persist or progress [26-33]. The clinical presentation of the disease followed a similar trend and evolution in both of our cases. Each twin had undergone a long history of slowly progressive pelvic pain from the point of intensity, developing bowel symptoms and dyschesia as time passed, with dyspareunia and change in stool diameter to the point of bowel stenosis and impending obstruction. All of this is consistent with the progressive nature of the disease reported in the existing literature.

Deep infiltrating endometriosis, contrary to being an estrogendependent disease, usually does not respond well to hormonal suppressive therapy. Adequate surgical excision of the lesions provides the best long-term results and symptomatic relief [3134] but surgical treatment of colorectal endometriosis has been quite controversial, and there is no consensus on whether the treatment should be conservative (shaving or discoid resection) or radical (segmental bowel resection and anastomosis) [35]. Lately, there has been a great deal of effort to advocate shaving as the treatment of choice, mainly because of higher early and late complications in segmental colorectal resection [36]. The efficacy of segmental colorectal resection is very debatable; data in this regard is controversial and, to a great deal, dependent upon the surgeon's experience $[37,38]$. Findings from histopathologic analysis of specimens from segmental bowel resection indicates that in $50 \%$ of cases there is a satellite lesion independent of the primary deep nodule.The deepest layer of the bowel wall containing endometrioticfoci at the primary lesion is in the submucosal layer in $70 \%$ of cases and the internal circular muscle layer in $30 \%$. Furthermore, persistent lesions are present in $50 \%$ of patients treated with discoid or shaving resection [39]. The latest systematic review of different surgical approaches to bowel and rectovaginal endometriosis showed that the complication rate is variable for conservative and radical treatment. Recurrence rate for shaving was reported at $22.2 \%$, for discoid resection $5.17 \%$, and in segmental resection $2.19 \%$; these rates were significantly different [37]. Positive bowel resection margins as well as age $<31$ years and body mass index $\geq 23 \mathrm{~kg} / \mathrm{m} 2$ appear to be important independent predictors of disease recurrence [40].

Our patients were managed according to the extent of their pathology, intensity of pelvic pain, and future fertility, thus minimising the chance of recurrence. Most importantly, patients' wishes and decisions regarding the type of clinical management to be pursued were incorporated, following proper counselling and explanation. It is crucial to keep in mind that there is currently no scientifically proven best treatment technique for bowel endometriosis, and comparison of different currently practised techniques is not possible. According to our current knowledge regarding the nature of disease pathology and its progression, recurrence should be as much a source of concern as complications for any given surgical technique. Resection of DIE should be global, not local, and limited to the bowel. Operations should be performed by an experienced surgeon and a well-organised team, in a multidisciplinary fashion. In summary, we present a case of advanced DIE in monozygotic twins; one of the first such cases to our knowledge. Our findings reiterate the importance of genetic factors in the aetiology of endometriosis. These cases also reinforce the importance of the following considerations for surgeons considering conservative treatment for colorectal endometriosis:

a) The extent of lesions cannot be assessed macroscopically with certainty.

b) Assessment of the depth of infiltration of endometriosis foci in the bowel requires an expert radiologist.

c) In the majority of rectal endometriosis nodules, the fibrosis which is the landmark for shaving does not surround, but follows behind the glandular epithelium and stroma.

d) More than $2 \mathrm{~cm}$ of bowel tissue must be removed from the main lesion to obtain clear margins in two thirds of patients.

e) Low rectal lesions $(<5-8 \mathrm{~cm}$ from the anal verge) are associated with a higher risk of complications.

f) A great majority of patients have been through previous surgeries and have a good chance of recovering and becoming pain-free.

\section{References}

1. Seracchioli R, Poggioli G, Pierangeli F, Manuzzi L, Gualerzi B, et al. (2007) Surgical outcome and long-term follow up after laparoscopic rectosigmoid resection in women with deep infiltrating endometriosis. BJOG 114(7): 889-895.

2. Wills HJ, Reid GD, Cooper MJ, Morgan M (2008) Fertility and pain outcomes following laparoscopic segmental bowel resection for colorectal endometriosis: a review. Aust N Z J Obstet Gynaecol 48(3): 292-295.

3. Kennedy S, Bergqvist A, Chapron C, D'Hooghe T, Dunselman G, et al. (2005) ESHRE guideline for the diagnosis and treatment of endometriosis. Hum Reprod 20(10): 2698-2704.

4. Nnoaham KE, Hummelshoj L, Webster P, De Cicco Nardone F, De Cicco Nardone C, et al. (2011) Impact of endometriosis on quality of life and work productivity: a multicenter study across ten countries. Fertil Steril 96(2): 366-373. 
5. Eskenazi B, Warner ML (1997) Epidemiology of endometriosis. Obstet Gynecol Clin North Am 24(2): 235-258.

6. Meuleman C, Vandenabeele B, Fieuws S, Spiessens C, Timmerman D, et al. (2009) High prevalence of endometriosis in infertile women with normal ovulation and normospermic partners. Fertil Steril 92(1): 68-74.

7. Treloar SA, O'Connor DT, O'Connor VM, Martin NG (1999) Genetic influences on endometriosis in an Australian twin sample. Fertil Steril 71(4): 701-710.

8. Simoens S, Dunselman G, Dirksen C (2012) The burden of endometriosis: costs and quality of life of women with endometriosis and treated in referral centres. Hum Reprod 27(5): 1292-1299.

9. Simpson JL, Elias S, Malinak LR, Buttram VC (1980) Heritable aspects of endometriosis. I. Genetic studies. Am J Obstet Gynecol 137(3): 327-331.

10. Stefansson H, Geirsson RT, Steinthorsdottir V, Jonsson H, Manolescu A, et al. (2002) Genetic factors contribute to the risk of developing endometriosis. Hum Reprod 17(3): 555-559.

11. Rahmioglu N, Nyholt DR, Morris AP, Missmer SA, Montgomery GW, et al. (2014) Genetic variants underlying risk of endometriosis: insights from meta-analysis of eight genome-wide association and replication datasets. Hum Reprod Update 20(5): 702-716.

12. Louis GM, Weiner JM, Whitcomb BW, Sperrazza R, Schisterman EF, et al. (2005) Environmental PCB exposure and risk of endometriosis. Hum Reprod 20(1): 279-285.

13. Parazzini F, Chiaffarino F, Surace M, Chatenoud L, Cipriani S, et al. (2004) Selected food intake and risk of endometriosis. Hum Reprod 19(8): 1755-1759.

14. Porpora MG, Medda E, Abballe A, Simone Bolli, Isabella De Angelis, et al. (2009) Endometriosis and organochlorinated environmental pollutants: a case-control study on Italian women of reproductive age. Environ Health Perspect 117(7): 1070-1075.

15. Fraser MA, Agarwal S, Chen I, Singh SS (2015) expert-guided transvaginal ultrasound in the diagnosis of endometriosis: a retrospective review. Abdom Imaging 40(3): 587-594.

16. Bazot M, Lafont C, Rouzier R, Roseau G, Thomassin-Naggara I, et al. (2009) Diagnostic accuracy of physical examination, transvaginal sonography, rectal endoscopicsonography, and magnetic resonance imaging to diagnose deep infiltrating endometriosis. Endometriosis 92(6): 1825-1833.

17. Goncalves MO, Podgaec S, Dias JA, Gonzalez M, Abrao MS (2010) Transvaginal ultrasonography with bowel preparation is able to predict the number of lesions and rectosigmoid layers affected in cases of deep endometriosis, defining surgical strategy. Hum Reprod 25(3): 665-671.

18. Chapron C, Santulli P, De Ziegler D, Noel JC, Anaf V, et al. (2012) Ovarian endometrioma: severe pelvic pain is associated with deeply infiltrating endometriosis. Hum Reprod 27(3): 702-711.

19. Acién P, Velasco I (2013) Endometriosis: A disease that remains enigmatic. ISRN Obstet Gynecol p.12.

20. Nisolle M, Donnez J (1997) Peritoneal endometriosis, ovarian endometriosis, and adenomyotic nodules of the rectovaginal septum are three different entities. Fertil Steril 68(4): 585-596.

21. Kamergorodsky G, Ribeiro PA, Galvao MA, Abrão MS, Donadio N, et al. (2009) Histologic classification of specimens from women affected by superficial endometriosis, deeply infiltrating endometriosis, and ovarian endometriomas. Fertil Steril 92(6): 2074-2077.

22. Sapkota Y, Attia J, Gordon SD, Henders AK, Holliday EG, et al. (2015) Genetic burden associated with varying degrees of disease severity in endometriosis. Mol Hum Reprod 21(7): 594-602.
23. Ding D, Liu X, Duan J, Guo SW (2015) Platelets are an unindicted culprit in the development of endometriosis: clinical and experimental evidence. Hum Reprod 30(4): 812-832.

24. Mehal WZ, Iredale J, Friedman SL (2011) Scraping fibrosis: expressway to the core of fibrosis. Nat Med 17(5): 552-553.

25. D’Hooghe TM, Bambra CS, Raeymaekers BM, Koninckx PR (1996) Serial laparoscopies over 30 months show that endometriosis in captive baboons (Papio anubis, Papio cynocephalus) is a progressive disease. Fertil Steril 65(3): 645-649.

26. Telimaa S, Ronnberg L, Kauppila A (1987) Placebo-controlled comparison of danazol and high-dose medroxyprogesterone acetate in the treatment of endometriosis after conservative surgery. Gynecol Endocrinol 1(4): 363-371.

27. Thomas EJ, Cooke ID (1987) Impact of gestrinone on the course of asymptomatic endometriosis. Br Med J (Clin Res Ed) 294(6567): 272274.

28. Cooke ID, Thomas EJ (1989) The medical treatment of mild endometriosis. Acta Obstet Gynecol Scand Suppl 150: 27-30.

29. Mahmood TA, Templeton A (1990) The impact of treatment on the natural history of endometriosis. Hum Reprod 5(8): 965-970.

30. Overton CE, Lindsay PC, Johal B, Collins SA, Siddle NC, et al. (1994) A randomized, double-blind, placebo-controlled study of luteal phase dydrogesterone (Duphaston) in women with minimal to mild endometriosis. Fertil Steril 62(4): 701-777.

31. Sutton CJ, Pooley AS, Ewen SP, Haines P (1997) Follow-up report on a randomized controlled trial of laser laparoscopy in the treatment of pelvic pain associated with minimal to moderate endometriosis. Fertil Steril 68(6): 1070-1074.

32. Harrison RF, Barry-Kinsella C (2000) Efficacy of medroxyprogesterone treatment in infertile women with endometriosis: a prospective, randomized, placebo-controlled study. Fertil Steril 74(1): 24-30.

33. Abbott J, Hawe J, Hunter D, Holmes M, Finn P, et al. (2004) Laparoscopic excision of endometriosis: a randomized, placebo-controlled trial. Fertil Steril 82(4): 878-884.

34. Koninckx PR, Ussia A, Adamyan L, Wattiez A, Donnez J (2012) Deep endometriosis: definition, diagnosis, and treatment. Fertil Steril 98(3): 564-571.

35. Abrão MS, Petraglia F, Falcone T, Keckstein J, Osuga Y, et al. (2015) Deep endometriosis infiltrating the recto-sigmoid: critical factors to consider before management. Hum Reprod Update 21(3): 329-339.

36. Roman H, Bridoux V, Tuech JJ, Marpeau L, da Costa C, et al. (2013) Bowel dysfunction before and after surgery for endometriosis. Am J Obstet Gynecol 209(6): 524-530.

37. Moustafa MM, Elnasharty MAA (2014) Systematic review of the outcome associated with the different surgical treatment of bowel and rectovaginal endometriosis. Gynecol Surg 11(1): 37-52.

38. Keckstein J, Wiesinger H (2005) Deep endometriosis, including intestinal involvement--the interdisciplinary approach. Minim Invasive Ther Allied Technol 14(3): 160-166.

39. Jinushi M, Arakawa A, Matsumoto T, Kumakiri J, Kitade M, et al. (2011) Histopathologic. J Minim Invasive Gynecol 18(1): 48-53.

40. Nirgianakis K, McKinnon B, Imboden S, Knabben L, Gloor B, et al. (2014) Laparoscopic management of bowel endometriosis: resection margins as a predictor of recurrence. Acta Obstet Gynecol Scand 93(12): 12621267. 


\begin{tabular}{ll} 
BIOMEDICAL & Assets of Publishing with us \\
RESEARCHES & Global archiving of articles \\
\hline & - Immediate, unrestricted online access \\
\end{tabular}

